\title{
Erythema Induratum of Bazin Accompanied by Atrophy of the Subcutaneous Fat
}

\author{
Hartati Purbo Dharmadji \\ Oki Suwarsa (1D \\ Endang Sutedja (i) \\ Miranti Pangastuti (D) \\ Hendra Gunawan (D) \\ Kristina Makarti
}

Department of Dermatology and Venereology, Faculty of Medicine, Universitas Padjadjaran-Dr. Hasan Sadikin Hospital, Bandung, Indonesia
Correspondence: Miranti Pangastuti Department of Dermatology and Venereology, Faculty of Medicine, Universitas Padjadjaran - Dr. Hasan Sadikin Hospital, Jl. Pasteur 38, Bandung, 4016I, West Java, Indonesia

Tel $+628 I 223114874$

Email pangastuti.miranti@gmail.com
Abstract: Erythema induratum of Bazin (EIB) is a predominantly lobular panniculitis with or without vasculitis due to Mycobacterium tuberculosis (MTB) infection. Atrophic scars may remain after EIB was healed. Rare cases of EIB showing sharp skin depression may mimic deep morphea and lupus profundus. A rare case of EIB was reported in a 35-year-old female with slightly tender erythematous-violaceous nodules and hyperpigmented depressed scars on both lower legs, resulting in asymmetrical lower leg shape. Histopathological examination showed lobular panniculitis without vasculitis and granulomatous inflammation consisted of caseous necrosis, epithelioid cells, and multinucleated giant cells. Polymerase chain reaction (PCR) from skin biopsy revealed deoxyribonucleic acid (DNA) of MTB. Tuberculin skin test (TST) was positive with a $20-\mathrm{mm}$ induration, and nuclear $99 \mathrm{mTc}-$ ethambutol scintigraphy was positive for tuberculosis infection in one-third distal portion of the left lower leg. Clinical improvements were shown after 6 months of antituberculosis therapy. EIB must be considered in patient with erythematous-violaceous nodules on lower leg, especially in endemic areas of tuberculosis infection since diagnosis can be difficult and occasionally, deceptive.

Keywords: erythema induratum of Bazin, Mycobacterium tuberculosis, panniculitis

\section{Introduction}

Panniculitis is defined as an inflammation of subcutaneous fat tissue with diverse etiologies. ${ }^{1,2}$ Erythema induratum of Bazin (EIB) is a predominantly lobular panniculitis associated with Mycobacterium tuberculosis (MTB) infection. $^{1,2}$ Vasculitis in some cases of EIB could not be demonstrated. Therefore, the presence of vasculitis should not be considered as a criteria for histopathologic diagnosis of EIB. ${ }^{3}$ The clinical manifestations of EIB include erythematous to violaceous nodules or deep plaques, tender or slightly tender, often progressing to ulcers, and heals with atrophic scar and hyperpigmentation. ${ }^{1,4}$ Although Indonesia is included in the high tuberculosis (TB) burden country, cases of EIB were rarely reported. ${ }^{1,4,5}$ Diagnosis of EIB is difficult since it can mimic other skin diseases, especially if there is no history of TB infections. ${ }^{1,4}$ Sharp skin depression in EIB is rarely reported. Deep morphea and lupus profundus are other types of panniculitis that can be difficult to distinguish clinically and histologically. ${ }^{6}$ Both may present with dermal and subcutaneous atrophy, particularly in chronic lesions. ${ }^{6,7}$ We present a case of EIB with clinical manifestations accompanied by sharply depressed skin mimicking deep morphea and lupus profundus. 


\section{Case}

A 35-year-old female presented to our clinic with slightly tender lumps and hyperpigmented depressed scars on both lower legs. The first lesions appeared ten years ago as erythematous macules and nodules. Some lesions became ulcerated and healed as hard hyperpigmented atrophic scars. Four months prior to consultation, new lumps appeared on both legs. There was no history of injuries, contact with TB patients, or symptoms of pulmonary or extrapulmonary TB. The patient was overweight while other aspects of general examination were within normal limit. Deep atrophy of the skin resulted in asymmetrical lower leg shape without movement disability (Figure 1). Varicose veins were observed on popliteal fossa (Figure 1). Dermatological examination revealed erythematous-violaceous, deep-seated nodules and macules, as well as hyperpigmented plaques on one-third distal portion of both lower legs. Some skin lesions showed superficial ulcers on deep skin atrophy covered with crusts and scales (Figure 1). Histopathological examination showed lobular panniculitis (Figure 2A) without vasculitis and granulomatous inflammation (Figure 2B) which consisted of caseous necrosis (red circle), epithelioid cells, and multinucleated giant cells (red arrow). There were no thickened collagen bundles, mucin deposition, and hyalinization. Direct immunofluorescence on skin lesion reveals no deposit of immunoglobulin or complement. The results of chest radiograph and sputum smear did not support the diagnosis of pulmonary TB. Tuberculin skin test (TST) was positive with a $20-\mathrm{mm}$ induration after 48 hours. Polymerase chain reaction (PCR) examination using GeneXpert MTB/RIF (Cepheid, Sunnyvale, CA, USA) on specimen obtained from skin biopsy was positive for deoxyribonucleic acid (DNA) of MTB. Nuclear 99mTc-ethambutol scintigraphy was positive for tuberculosis infection in the one-third distal

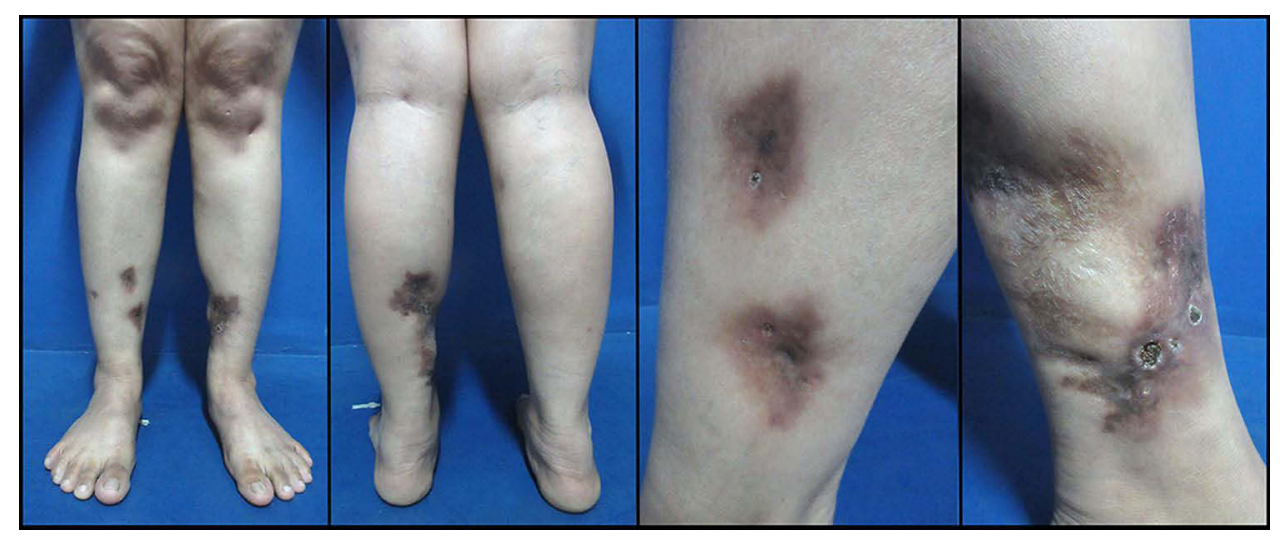

Figure I Clinical image on day I. Erythematous to violaceous, well-defined, deep-seated nodules and macules mostly over the distal-third of the lower legs. Some of the lesions showed superficial ulcer covered with crusts and scales.

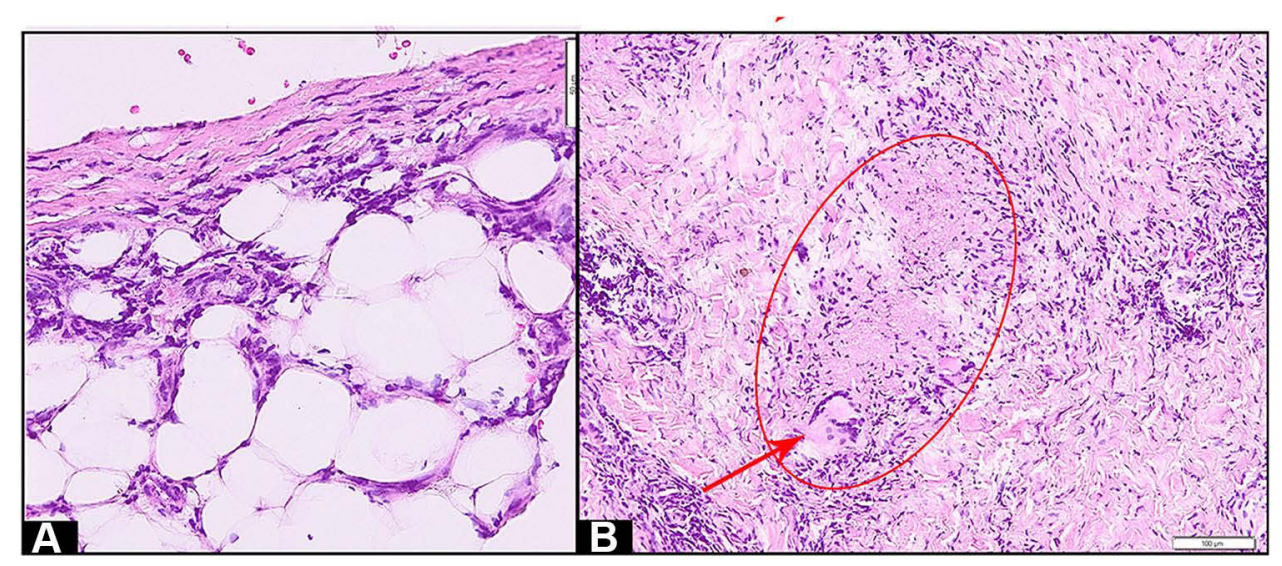

Figure 2 Histopathology of erythema induratum of Bazin. Skin biopsy shows lobular panniculitis (A), granulomatous inflammation consisted of caseous necrosis (red circle), epithelioid cell proliferation, and multinucleated giant cells (red arrow) (B). (Hematoxylin-eosin stain; original magnification, 200x (A), and I00x (B), respectively). 


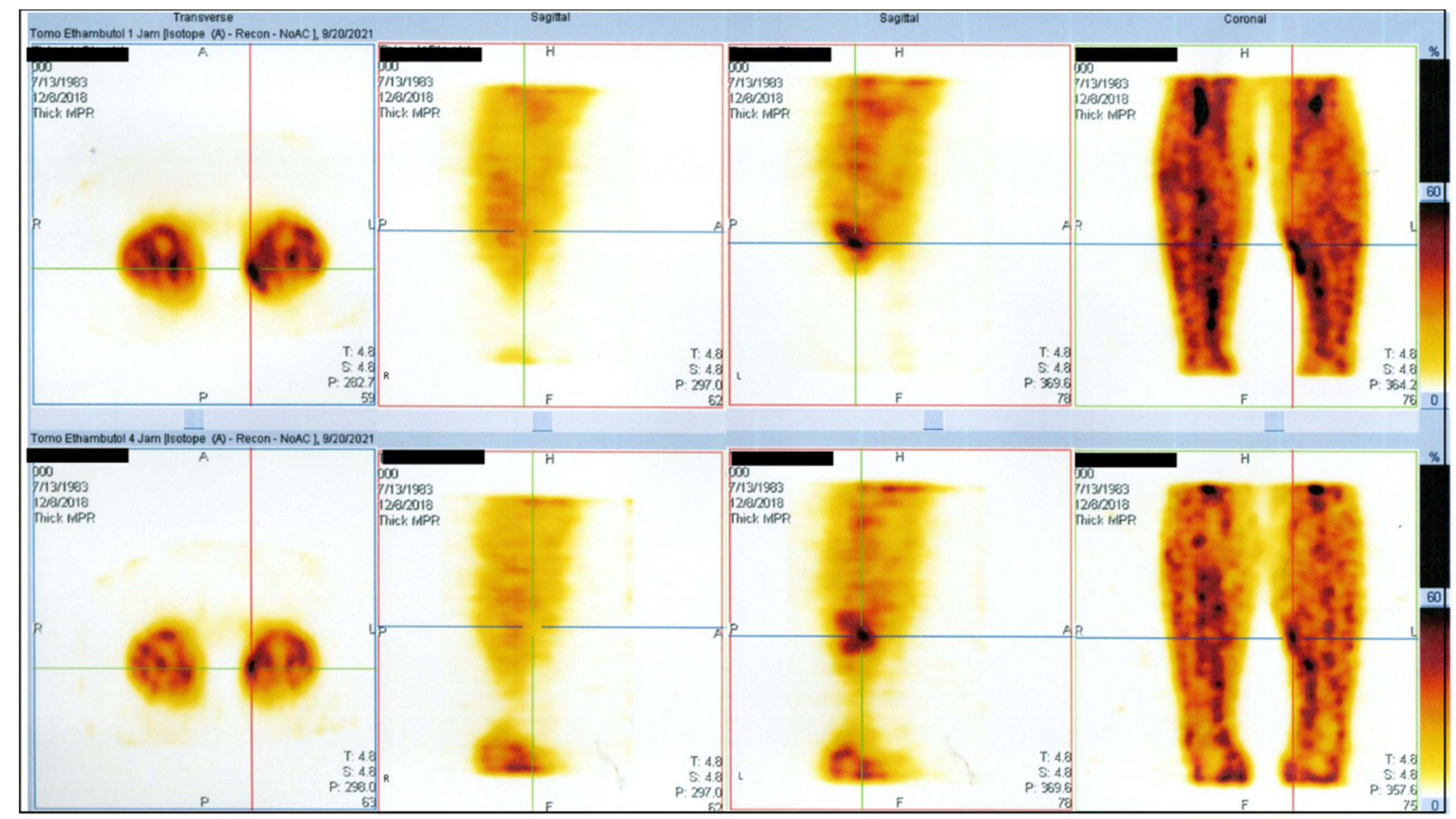

Figure 3 Nuclear $99 \mathrm{mTc}$-ethambutol scintigraphy shows increased pathological radioactivity imaging in the distal-third of the left lower leg at 4 hours postradiopharmaceuticals injection.

portion of the left lower leg (Figure 3). Based on those results, the diagnosis of EIB was established. The patient was therefore treated with fixed-dose combination (FDC) of anti-tuberculosis drugs. After 6 months of treatment, the nodules regressed and healed, and no new skin lesions occurred (Figure 4).

\section{Discussion}

Deep morphea and lupus profundus are variants of inflammatory panniculitis which are difficult to distinguish clinically. ${ }^{6}$ Deep morphea is characterized by an inflammatory ill-defined plaque or nodule with symmetrical distribution that typically affects the legs, forearms, and trunk. The skin typically feels thickened due to attachment to the fascia and underlying muscles. ${ }^{6,8}$ In the inflammatory stage, morphea begins as an erythematous plaque or patches. Later, in the sclerotic stage, sclerosis develops centrally with surrounding hyperpigmentation. ${ }^{8}$ Over the course of months to years, the sclerotic plaque become atrophic with hypopigmentation or hyperpigmentation (atrophic stage) that manifested as cigarette paper wrinkling (papillary dermis), cliff drop (dermal), or deep indentations

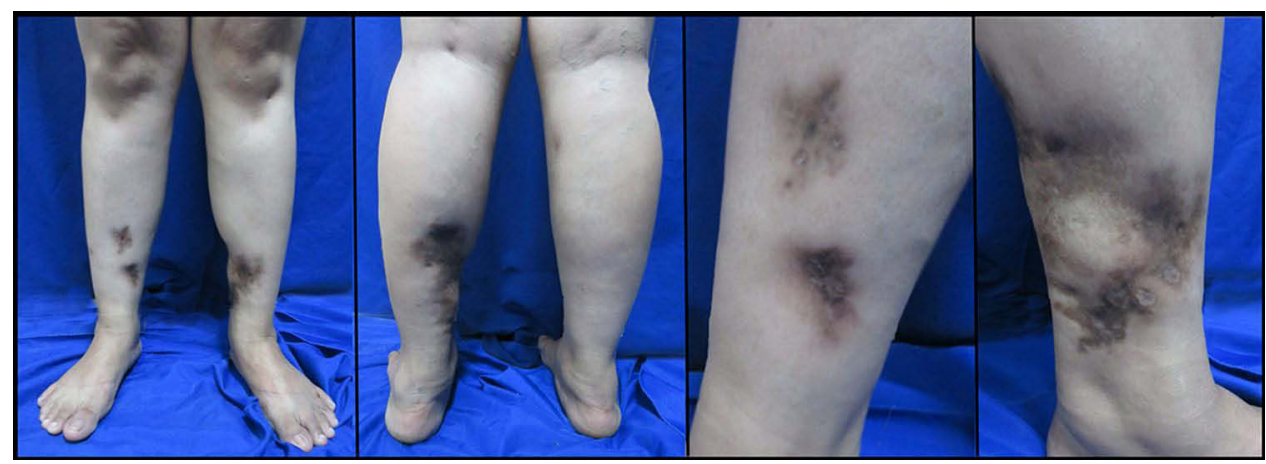

Figure 4 Clinical image after 6 months of anti-tuberculosis therapy. Cutaneous nodules started to regress, ulcer and crusts healed with atrophic scar and post-inflammatory hyperpigmentation. 
(subcutis or deeper atrophy). ${ }^{8}$ Lupus profundus is a distinct variant of cutaneous lupus that is generally classified as a form of chronic cutaneous lupus erythematosus. ${ }^{7}$ Lupus profundus is clinically characterized by tender, subcutaneous nodules involving the proximal extremities, trunk, face, and scalp. ${ }^{6,7}$ The disease resolves leaving a depressed atrophic scar, with similar presentation to deep morphea. ${ }^{7}$ Clinical features of EIB include multiple nodules or deep plaques, concentrated on the one-third distal portion of the lower leg. Lesions may be tender, slightly tender, or tender only on pressure. ${ }^{3,4}$ The nodules may develop focal ulceration, which later healed with depressed scars and pigmentation. ${ }^{4}$ The disease course is protracted and recurrent over years, even decades. There are rare EIB cases showing sharp skin depression as part of their skin manifestations. EIB commonly occurred in adult women with 9:1 female-to-male ratio. It is correlated with obesity and venous insufficiency. ${ }^{1,4}$

In our case, deep atrophy and hardened skin lesions were part of the clinical manifestations that resulted in asymmetrical shape of the lower leg, resembling deep morphea and lupus profundus. However, this patient had slightly tender multiple erythematous to violaceous deepseated nodules on one-third distal portion of both lower legs, which became ulcerated and healed with hyperpigmentation and atrophic scars. The patient was an overweight adult woman who had varicose veins in both lower legs. Therefore, we consider EIB as one of the differential diagnosis.

The histopathological features of EIB include lobular or diffuse septolobular panniculitis, vasculitis, as well as granulomatous inflammation with caseous necrosis, epithelioid cells, and multinucleated giant cells. ${ }^{1,4}$ Segura et $\mathrm{al}^{3}$ reported that $9.9 \%$ of biopsy specimens in EIB cases failed to demonstrate clear-cut histopathologic features of vasculitis. Deep morphea and lupus profundus can be difficult to distinguish histologically. ${ }^{6}$ The histopathological features of deep morphea are septal panniculitis without vasculitis, thickened collagen bundles and hyalinization. ${ }^{2,8,9}$ The histopathological findings of lupus profundus are lobular or mixed panniculitis with lymphocytic infiltrate, mucin deposition, and hyaline fat necrosis. ${ }^{7}$ Lupus profundus shows positive direct immunofluorescence results, whereas deep morphea shows negative results. $^{6}$ Our patient showed histopathological features consistent with that of EIB.
Detection of MTB in the skin lesions of subjects with tuberculin test proved that mycobacterial components were involved in the pathogenesis. ${ }^{10}$ A study by Baselga et al ${ }^{11}$ on 65 EIB patients found MTB DNA in $77 \%$ of biopsy specimens using PCR. Another study by Magalhães et al ${ }^{12}$ on 54 EIB patients found positive TST with an induration of $>15 \mathrm{~mm}$ diameter in $79.1 \%$ of subjects. In the current case, MTB DNA from skin lesion was detected by PCR examination and TST was positive with $20 \mathrm{~mm}$ induration diameter. Indonesia is a country with high TB burden, ${ }^{5}$ and it is important to prove extrapulmonary $\mathrm{TB}$ infection in EIB cases. ${ }^{4,10}$ The current patient's chest radiograph and sputum smear did not support the diagnosis of pulmonary TB. Nuclear 99mTc-ethambutol scintigraphy is a diagnostic imaging technique that can help diagnose and determine the location of MTB infection. This examination for extrapulmonary TB has a sensitivity and specificity of $95.5 \%$ and $77.8 \%$, respectively. ${ }^{13}$ The location of TB infection in the current case was obtained based on increased pathological radioactivity imaging in one-third distal portion of the left lower leg observed 4 hours after injection of $99 \mathrm{mTc}$-ethambutol.

Patients with underlying MTB infection, as confirmed through a positive TST, with the presence of MTB DNA on skin lesions, supported with other investigations, must be treated with antituberculosis agents. ${ }^{1,4}$ Our patient was treated with FDC of antituberculosis agents. After 6 months on antituberculosis therapy, her skin lesions showed positive response and significant improvement.

The diagnosis criteria for EIB are as follows: patients with chronic inflammatory nodules on the leg, evidence of MTB infection (markedly positive TST or active infection with MTB), and responsive features after anti-tuberculosis therapy. ${ }^{4,10}$ In our patient, the diagnosis of EIB was supported by appropriate clinical features, positive findings on histopathology, TST, PCR of MTB, nuclear 99mTcethambutol scintigraphy, and responsiveness after antituberculosis therapy.

\section{Conclusion}

Clinical manifestation of EIB can mimic another skin disease such as deep morphea and lupus profundus, so diagnosis can be difficult if there is no history of TB infection. EIB must be considered as a differential diagnosis when encountering a woman with tender or slightly tender nodules on the lower legs in endemic areas of tuberculosis infection. 


\section{Abbreviations}

EIB, Erythema induratum of Bazin; MTB, Mycobacterium tuberculosis; TB, Tuberculosis; TST, Tuberculin skin test; PCR, Polymerase chain reaction; DNA, Deoxyribonucleic acid; FDC, Fixed-dose combination.

\section{Ethical Approval}

This study was conducted in compliance with the Declaration of Helsinki, Good Clinical Practices, local regulatory requirements, and was approved by the Medical Ethics Committee of Hasan Sadikin General Hospital Bandung (approval number: LB.02.01/X.6.5/323/2020).

\section{Consent for Publication}

The patient signed informed consent forms. She also signed forms giving consent for the use of case details and images for publication and for scientific purposes.

\section{Acknowledgments}

The authors would like to thank the staff of Department of Dermatology and Venereology, Faculty of Medicine, Universitas Padjadjaran, Bandung, West Java, Indonesia.

\section{Author Contributions}

All authors made a significant contribution to the work reported, whether that is in the conception, study design, execution, acquisition of data, analysis, and interpretation, or in all these areas; took part in drafting, revising or critically reviewing the article; gave final approval of the version to be published; have agreed on the journal to which the article has been submitted; and agree to be accountable for all aspects of the work.

\section{Disclosure}

The authors report no conflicts of interest in this work.

\section{References}

1. Lake EP, Worobec SM, Aronson IK, et al. Panniculitis. In: Kang S, Amagai M, Bruckner AL, Enk AH, Margolis DJ, McMichael AJ, editors. Fitzpatrick Dermatology. 9th ed. New York: McGraw-Hill; 2019:1251-1262.

2. Gupta P, Saikia UN, Arora S, De D, Radotra BD. Panniculitis: a dermatopathologist's perspective and approach to diagnosis. Indian $J$ Dermatopathol Diagn Dermatol. 2016;3(2):29. doi:10.4103/2349-6029.195224

3. Segura S, Pujol RM, Trindade F, Requena L. Vasculitis in erythema induratum of Bazin: a histopathologic study of 101 biopsy specimens from 86 patients. J Am Acad Dermatol. 2008;59(5):839-851. doi:10.1016/j.jaad.2008.07.030

4. Cho KH. Inflammatory nodules of the leg. Ann Dermatol. 2012;24 (4):383-392. doi:10.5021/ad.2012.24.4.383

5. Raviglione M, Sulis G. Tuberculosis 2015: burden, challenges and strategy for control and elimination. Infect Dis Rep. 2016;8(2). doi:10.4081/idr.2016.6570

6. Mangold AR, Costello CM, Cumsky HJ, DiCaudo DJ, Griffing WL, Pittelkow MR. Systemic scleroderma and lupus panniculitis with atypical clinical features: a case report and comprehensive review. JAAD Case Rep. 2018;4(8):789-793. doi:10.1016/j.jdcr.2018.03.022

7. Hansen CB, Callen JP. Connective tissue panniculitis: lupus panniculitis, dermatomyositis, morphea/scleroderma. Dermatol Ther. 2010;23(4):341-349. doi:10.1111/j.1529-8019.2010.01334.x

8. Cyrus N, Jacobe HT. Morphea and lichen sclerosus. In: Kang S, Amagai M, Bruckner AL, Enk AH, Margolis DJ, McMichael AJ, editors. Fitzpatrick Dermatology. 9th ed. New York: McGraw-Hill 2019:1106-1116

9. Bielsa I, Ariza A. Deep morphea. Semin Cutan Med Surg. 2007;26 (2):90-95. doi:10.1016/j.sder.2007.02.005

10. $\mathrm{Xu} \mathrm{L}$, Flattery D, McCabe R. Not the usual suspect: a case of erythema induration of Bazin in an urban primary care clinic. $J$ Community Hosp Intern Med Perspect. 2015;5(6):29342. doi:10.3402/jchimp.v5.29342

11. Baselga E, Margall N, Barnadas MA, Coll P, de Moragas JM. Detection of Mycobacterium tuberculosis DNA in lobular granulomatous panniculitis (erythema induratum-nodular vasculitis). Arch Dermatol. 1997;133(4):457-462. doi:10.1001/ archderm.1997.03890400057008

12. Magalhães TS, Dammert VG, Samorano LP, Litvoc MN, Nico MM. Erythema induratum of Bazin: epidemiological, clinical and laboratorial profile of 54 patients. $J$ Dermatol. 2018;45(5):628-629. doi:10.1111/1346-8138.14260

13. Kartamihardja AH, Kurniawati Y, Gunawan R. Diagnostic value of $99 \mathrm{~m}$ Tc-ethambutol scintigraphy in tuberculosis: compared to microbiological and histopathological tests. Ann Nucl Med. 2018;32 (1):60-68. doi:10.1007/s12149-017-1220-1
International Medical Case Reports Journal

\section{Publish your work in this journal}

The International Medical Case Reports Journal is an international, peer-reviewed open-access journal publishing original case reports from all medical specialties. Previously unpublished medical posters are also accepted relating to any area of clinical or preclinical science. Submissions should not normally exceed 2,000 words or 4 published pages including figures, diagrams and references. The manuscript management system is completely online and includes a very quick and fair peer-review system, which is all easy to use. Visit http://www.dovepress.com/testimonials.php to read real quotes from published authors. 